de Mecánica Computacional

Mecánica Computacional Vol XXIX, págs. 2947-2947 (resumen)

Eduardo Dvorkin, Marcela Goldschmit, Mario Storti (Eds.)

Buenos Aires, Argentina, 15-18 Noviembre 2010

\title{
PHASE STABILITY AND TRANSFORMATIONS IN NITI FROM DENSITY FUNCTIONAL THEORY CALCULATIONS
}

\section{Alejandro Strachan and Karthik Guda}

Purdue University, United States, \{strachan; kgudavis\}@purdue.edu

\begin{abstract}
We use density functional theory to characterize various crystalline phases that govern the response of NiTi alloys including shape memory and super elasticity. We also investigated possible transition pathways between the various phases and the energetics involved. We predict a new phase of NiTi, denoted B19", which is involved in the transition between B19' (the experimentally observed martensite phase) and BCO (the theoretically predicted ground state). B19" is monoclinic and can exhibit shape memory. Furthermore, its presence reduces the internal stress required to stabilize the experimentally observed B19' phase. We also characterize the surface energy of the various phases and show how reducing the specimen size to the nanoscale affects the relative stability of the phases and, consequently, their mechanical response.
\end{abstract}

